\title{
Erratum to: Spatial analysis of root hemiparasitic shrubs and their hosts: a search for spatial signatures of above- and below-ground interactions
}

\author{
Bjørn Dueholm (1D) David Bruce $\cdot$ Philip Weinstein • \\ Susan Semple $\cdot$ Birger Lindberg Møller $\cdot$ Jacob Weiner
}

Published online: 2 December 2016

(C) Springer Science+Business Media Dordrecht 2016

\section{Erratum to: Plant Ecol \\ DOI 10.1007/s11258-016-0676-8}

The original publication of the article includes an error in Figs. 2 and 3. The correct version of Figs. 2 and 3 are provided in this erratum.

The online version of the original article can be found under doi:10.1007/s11258-016-0676-8.

B. Dueholm $(\bowtie) \cdot$ P. Weinstein · S. Semple School of Pharmacy and Medical Sciences, University of South Australia, GPO Box 2471, Adelaide, SA 5001,

Australia

e-mail: bjorn.dueholm@mymail.unisa.edu.au

B. Dueholm · B. L. Møller · J. Weiner

Department of Plant and Environmental Sciences,

University of Copenhagen, Thorvaldsensvej 40,

1871 Frederiksberg C, Denmark

D. Bruce

School of Natural and Built Environments, University of South Australia, GPO Box 2471, Adelaide, SA 5001,

Australia

P. Weinstein

School of Biological Sciences, University of Adelaide,

Adelaide, SA 5005, Australia 
Fig. 2 Density

investigations of A. ligulata in relation to root hemiparasitic shrubs. a Above-ground biomass densities $\left(\mathrm{m}^{3}\right.$ per square metre) of A. ligulata shrubs within the study site. Red discs show locations of the root hemiparasitic shrubs and black discs show the locations of the A. ligulata shrubs. b The intensity estimates (black lines) of unmarked A. ligulata (left panel), and marked $A$. ligulata (right panel) using the above-ground volume of each individual shrub.

Additionally, 95\%

confidence intervals are included (grey bands). (Color figure online)

\section{a}
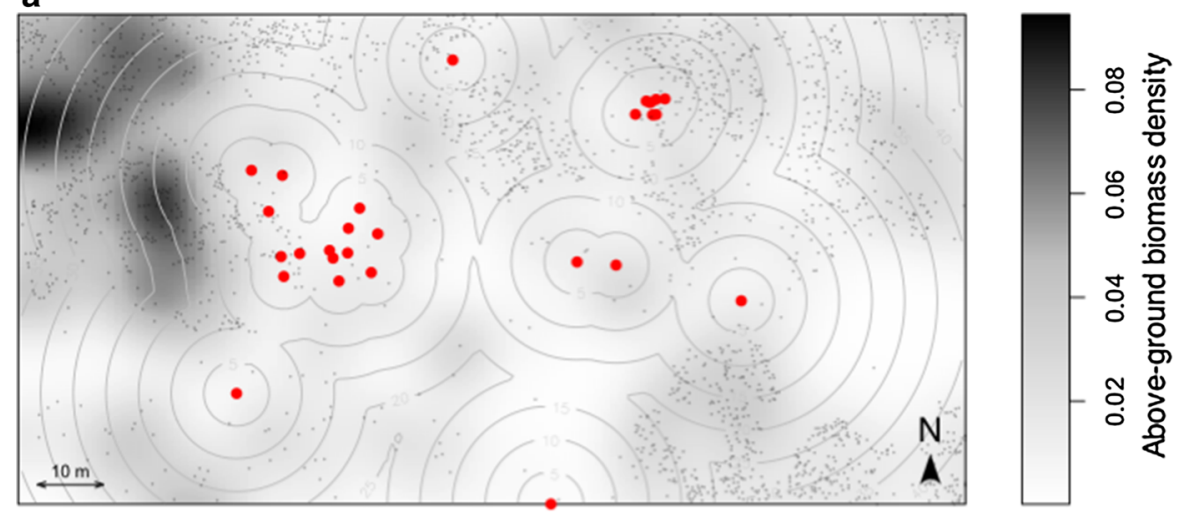

b

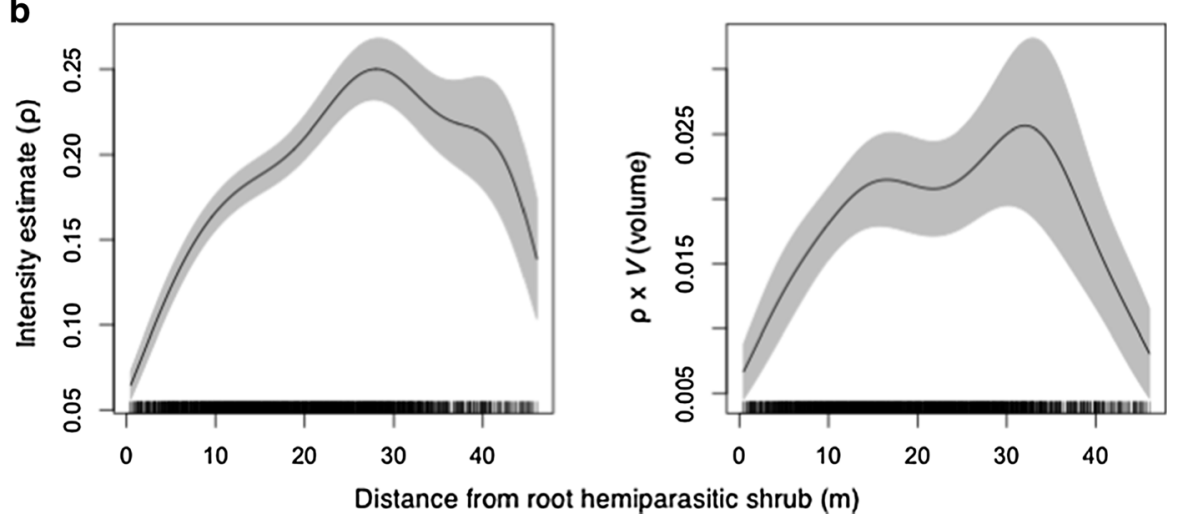




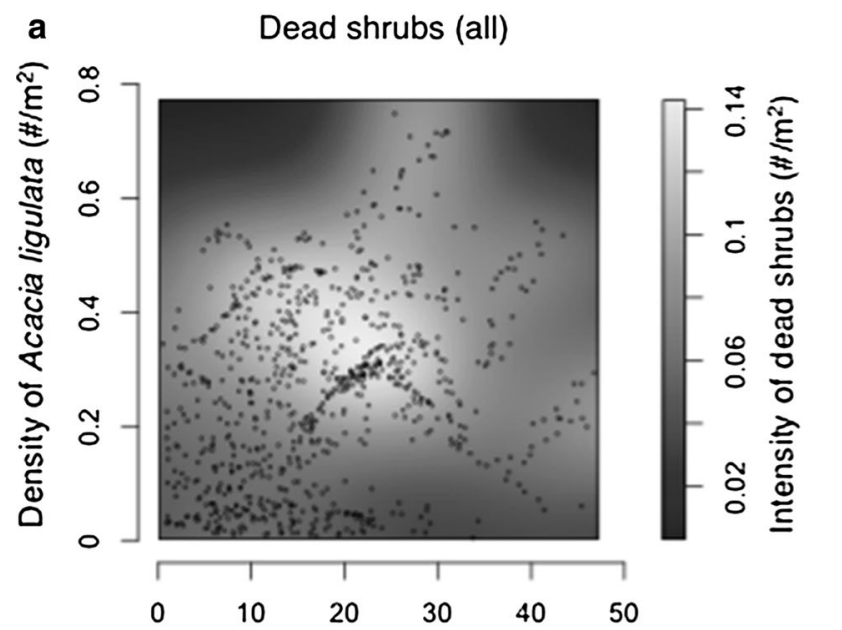

Dead shrubs (excluding Cassytha-infected)

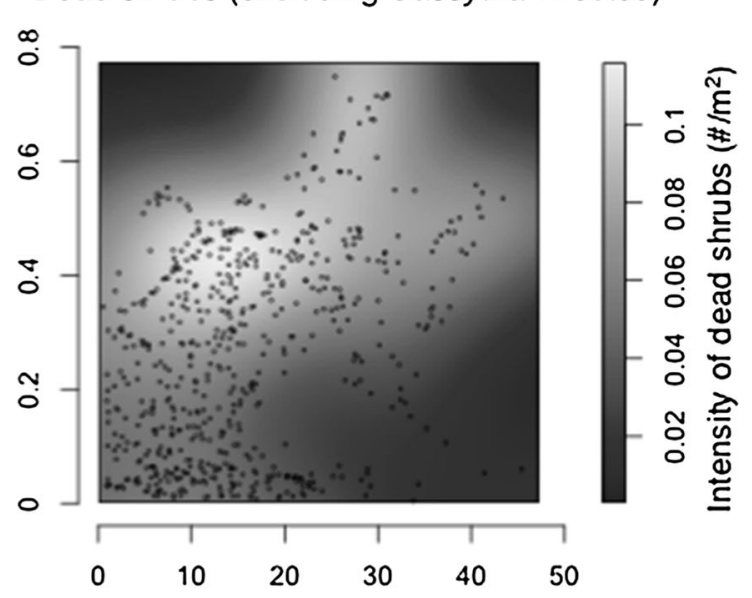

b

Distance from root hemiparasitic shrub $(\mathrm{m})$

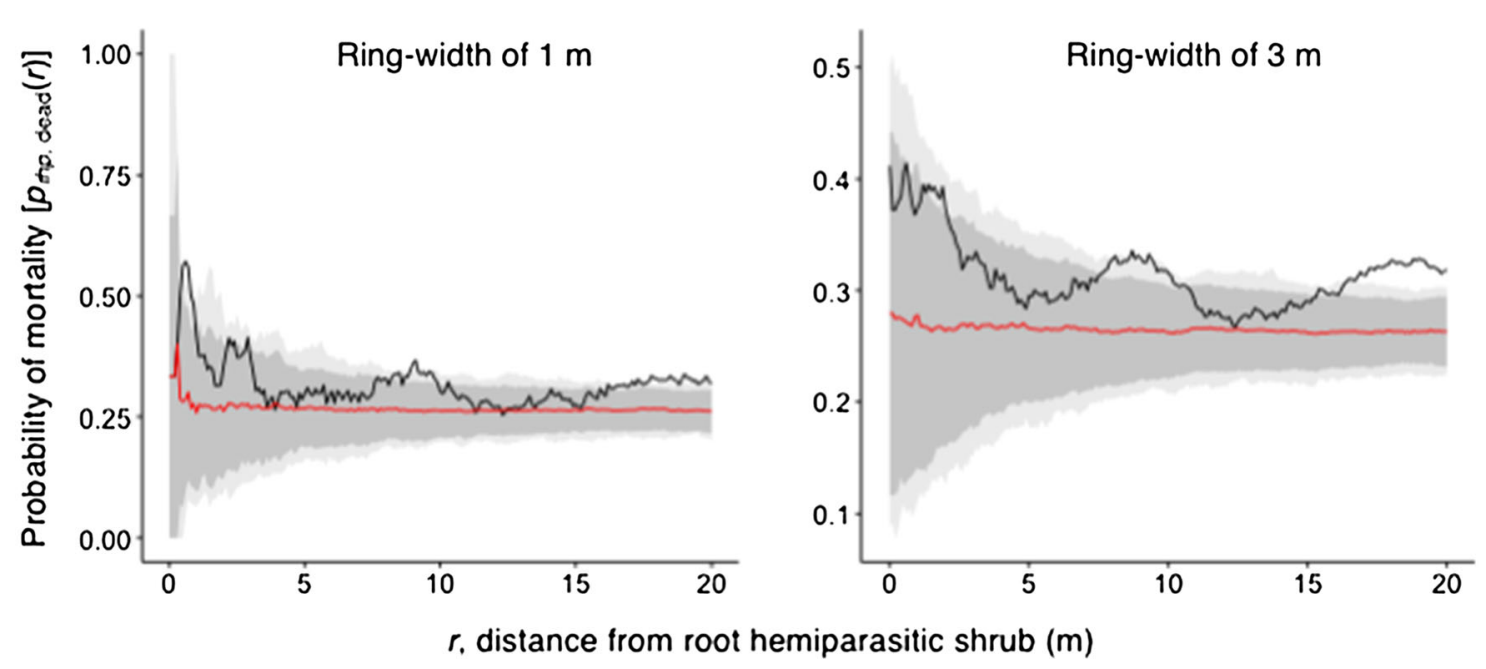

Fig. 3 Mortality investigations. a Estimated intensities of dead shrubs using either all shrubs (left panel) or those excluding $C$. melantha haustorial remnants (right panel) as a function of both distance from root hemiparasitic shrubs and density of $A$. ligulata. b Trivariate random labelling using the root hemiparasites as the antecedent focal pattern (subscript rhp) in order to investigate whether the probability of mortality of A. ligulata individuals (dead shrubs) within a 20-m distance from the root hemiparasites was higher than under randomness. Simulation envelopes of random labelling were created by shuffling the qualitative marks ('dead') over the locations of dead and live $A$. ligulata shrubs (light-grey band 5th highest and lowest simulation values, dark-grey band 25th highest and lowest simulation values). The red lines are the means of the simulation results representing complete spatial randomness (i.e. $p_{\text {rhp,dead }}(-$ $r) \approx 0.26$ ). Two different ring widths ( $1 \mathrm{~m}$, left panel; $3 \mathrm{~m}$, right panel) were used for estimating the intensity normalised neighbourhood densities. (Color figure online) 